\title{
Effect of clopidogrel discontinuation at 1 year after drug eluting stent placement on soluble CD40L, P-selectin and C-reactive protein levels: DECADES (Discontinuation Effect of Clopidogrel After Drug Eluting Stent): a multicenter, open-label study
}

\author{
Joanna J. Wykrzykowska · Ascan Warnholtz • Peter de Jaeger • Nick Curzen • \\ Keith G. Oldroyd · Jean Philippe Collet · Jurrien M. Ten Berg · Tessa Rademaker • \\ Dick Goedhart · Jurgen Lissens · Peter-Paul Kint · Patrick W. Serruys
}

Published online: 6 June 2009

(c) The Author(s) 2009. This article is published with open access at Springerlink.com

\begin{abstract}
Antiplatelet therapy with clopidogrel has been shown to reduce major adverse cardiac events in acute coronary syndromes and after percutaneous interventions. This effect is not only due to its anti-platelet effect but also possibly due to an anti-inflammatory effect. The effect of clopidogrel cessation after one year of therapy on markers of inflammation has been investigated in diabetics and showed an increase in platelet aggregation as well as hsCRP and surface P-selectin levels. This was
\end{abstract}

J. J. Wykrzykowska · P. de Jaeger · P. W. Serruys ( $\square)$ Interventional Cardiology, Thoraxcenter, Erasmus MC, University Medical Center, 's Gravendijkwal 230, 3015CE Rotterdam, The Netherlands

e-mail: p.w.j.c.serruys@erasmusmc.nl

A. Warnholtz

Klinikum der Johannes Mainz, Mainz, Germany

N. Curzen

Southampton General Hospital, Southampton, UK

K. G. Oldroyd

Western Infirmary, Glasgow, UK

J. P. Collet

Group Pitie-Salpetriere Hospital Cardiology Institute,

Paris, France

J. M. Ten Berg

Sint Antonius Ziekenhuis, Utrecht, The Netherlands

T. Rademaker - D. Goedhart · P.-P. Kint

Cardialysis BV, Rotterdam, The Netherlands

J. Lissens

Bristol-Myers Squibb, International Company,

Bruxelles, Belgium an exploratory multicenter prospective open-label single arm study of 98 non-diabetic patients who had received one or more drug eluting stents and were coming to the end of their 12 months course of clopidogrel therapy. The effect of clopidogrel cessation on expression of biomarkers: sCD40L, soluble P-selectin and hsCRP was measured right before clopidogrel cessation (day 0 ), and subsequently at $1,2,3$ and 4 weeks after drug withdrawal. A median increase in sCD40L expression from 224 to $324.5 \mathrm{pg} / \mathrm{ml}$ was observed between baseline and 4 weeks after clopidogrel cessation, which corresponded to a $39 \%$ mean percent change based on an ANCOVA model $(P<0.001)$. Over the 4 weeks observation period the change in sCD40L expression correlated weakly with soluble P-selectin levels (at 4 weeks Spearman's correlation coefficient $=0.32 ; P=0.0024)$. Increase in P-selectin expression from baseline was statistically significant at week 1 and 2. Conversely, hsCRP level decreased by $21 \%$ at 1 week $(P=0.008)$ and was still reduced by $18 \%$ by 4 weeks $(P=0.062)$. The change in sCD40L expression appeared to vary with the type of drug eluting stent. Patients treated with drug eluting stents at 1 year after implantation display significant increase in sCD40L and decrease in hsCRP after clopidogrel cessation. Further studies should elucidate if this increase in sCD40L levels reflects solely the removal of the inhibitory effects of clopidogrel on platelet activity or rather an increase in pro-inflammatory state. The latter hypothesis may be less likely given decrease in hsCRP levels. Randomized studies are urgently needed to establish potential link of clopidogrel discontinuation and vascular outcomes.

Keywords Clopidogrel cessation - Platelet inhibition · Inflammation · Drug eluting stent 


\section{Introduction}

Addition of clopidogrel to aspirin results in reduction in platelet reactivity [1]. This effect is particularly important in patients that have been treated with percutaneous coronary angioplasty and stenting [2]. Those who are nonresponders to clopidogrel may be at higher risk of adverse events. Similarly, patients who interrupt their clopidogrel therapy may be at risk of major adverse cardiovascular event, among them stent thrombosis. Late stent thrombosis has been shown to occur after drug eluting stent implantation at a rate of $0.6 \%$ per year for up to 4 years [3]. While the pathogenesis of stent thrombosis is likely multifactorial, from stent malapposition (initial and acquired), geographic miss [4], through polymer-induced inflammation and incomplete tissue coverage [5], it has also been suggested that discontinuation of dual anti-platelet therapy plays a role in a large proportion of cases [6, 7]. Increased pro-coagulability after withdrawal of platelet inhibition is likely a major factor, as incomplete platelet inhibition has emerged as a significant risk factor for major adverse cardiac events [8-11]. What has also emerged as a possible mechanism is a potential increase in inflammation after clopidogrel cessation. The role of clopidogrel, as well as GP IIb/IIIa inhibitors, in modulating inflammation has been documented in multiple studies in the context of acute coronary syndrome and percutaneous coronary intervention [12-17]. The effect of clopidogrel cessation on inflammatory marker expression has not been investigated to date, other than in a small cohort of 54 diabetic patients [8]. In this study, platelet aggregation as well as hsCRP levels and surface platelet P-selectin levels increased significantly at 4 weeks after cessation of clopidogrel. It is not known whether cessation of treatment in non-diabetic patients also leads to pro-inflammatory marker upregulation and, if so, whether this could cause an increase in late stent thrombosis in a proportion of patients. In this exploratory study, we set out to describe the changes in the levels inflammatory markers sCD40L, P-selectin and hsCRP within 4 weeks of clopidogrel withdrawal in a 100 non-diabetic patients treated with stable doses of aspirin and statin.

\section{Methods}

Study design

The Discontinuation Effect of Clopidogrel After Drug Eluting Stent (DECADES) trial was an exploratory, multicenter, open-label, single-arm study to determine the effects of withdrawing clopidogrel therapy 12 months after implantation of DES on markers of inflammation and platelet activation in a non-diabetic population. The trial took place between 31 October 2007 and 30 June 2008 and was conducted at 6 sites in 4 European countries. The trial was approved by the institutional review board/independent ethics committee at every study centre. Written informed consent for trial participation was obtained from each patient. The trial was conducted in line with the guidelines of the current amendment to the Declaration of Helsinki, in accordance with the International Conference on Harmonization of Good Clinical Practice and applicable regulatory requirements.

\section{Study population}

Patients older than 18 , who had received $\geq 1$ DES of any type, were receiving statin and low-dose aspirin therapy, and were coming to the end of 12 months of therapy with clopidogrel $75 \mathrm{mg} /$ day, were eligible to participate in the trial. Current medication doses (including aspirin and statins) must have been stable for 3 months. Patients were excluded from the study if they had a clinical history of diabetes mellitus, had uncontrolled hypertension (systolic blood pressure $>$ $180 \mathrm{mmHg}$ or diastolic $\mathrm{BP}>100 \mathrm{mmHg}$ ), were intolerant of (or had contraindications to) aspirin or statins. The following patients were also excluded: those who had used within the previous 3 months or were currently using oral anticoagulants, dipyridamole or oral glucocorticoids; or were taking another investigational study medication or had taken an investigational study medication within the previous 30 days of the enrolment visit. Use of antiplatelet agents, other than stable doses of low-dose aspirin, was prohibited during the study.

\section{Study protocol}

After obtaining written informed consent, eligible patients were enrolled in the study. At this time, therapy with clopidogrel $75 \mathrm{mg} /$ day was stopped, and stable dosages of statins and low-dose aspirin continued. The aim of the study was to evaluate the discontinuation effect of clopidogrel on biomarkers by taking blood samples prior to or on the day of

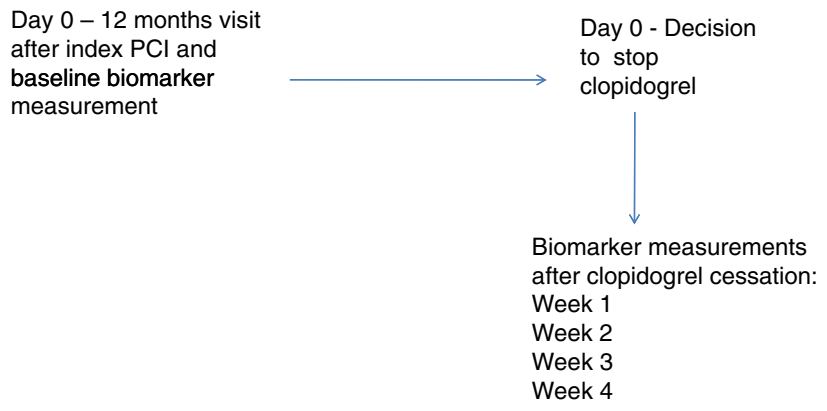

Fig. 1 Study flow chart 
discontinuation of clopidogrel (baseline), and then at followup visits at the end of weeks 1, 2, 3 and 4 (Fig. 1).

Safety was evaluated during the entire study period by reporting any adverse events and serious adverse events. At each follow-up visit patients were asked if they had experienced any symptoms since clopidogrel withdrawal.

\section{Biomarker assessments}

Blood samples were obtained by direct venipuncture by an experienced phlebotomist. The primary endpoint was the mean change from baseline to week 4 in levels of sCD40L. Secondary endpoints included changes from baseline in plasma soluble P-selectin and hsCRP levels. All blood samples were frozen and sent for analysis to a central laboratory (Quintiles Limited, Livingston, UK). sCD40L, soluble P-selectin and hsCRP levels were analysed.

\section{Inflammation markers}

The blood samples collected for immunoassays were centrifuged at $1400 \mathrm{~g}$ for $10 \mathrm{~min}$. Measurements of hsCRP, CD40L and P-selectin were performed in duplicate by enzyme-linked immunosorbent assay using commercially available kits from R\&D Systems (Minneapolis, MN) for sCD40L and P-selectin and DPC Immulite for hsCRP (Block Scientific, Holbrok, NJ).

\section{Statistical methods}

This was an exploratory study and therefore data on the effect of withdrawing clopidogrel on sCD40L were not available for this study population. The sample size was not based on power considerations. Analyses were performed in the clopidogrel cessation population. All patients signed informed consent. The primary and secondary endpoint variables were evaluated using an ANCOVA model with baseline as covariate and investigative site as the main effect. As all three biomarkers showed a lognormal distribution at week 4, all ANCOVA analyses were performed on log-transformed values (using the natural logarithm transformation). The least-square mean was computed using a weighting scheme based on the actual sample sizes at each site. Data in the pre-specified analysis are presented as mean change from baseline, with $95 \%$ confidence intervals (CI). For all changes from baseline analyses, only subjects with at least a baseline and one post-clopidogrel withdrawal measurement were included. No imputation technique was used to attempt to account for missing data. Demographic and baseline characteristics, as well as safety data, were summarized using descriptive statistics, medians and interquartile ranges. Analyses were performed using the SAS 8.2 statistical package.
As post-hoc analysis, correlation between P-selectin levels and $\mathrm{SCD} 40 \mathrm{~L}$ was performed. In addition, the effect of the type of DES on the change from baseline in levels of SCD40L was assessed using an ANCOVA model with baseline as covariate and type of DES as the main effect.

\section{Results}

\section{Study population}

A total of 103 patients were enrolled in the study and 98 patients completed the protocol. Two patients failed to have baseline biomarker measurements. Two patients withdrew their consent and one patient no longer met the criteria for enrolment after baseline evaluation. Baseline characteristics are summarized in Table 1A. They were predominantly male with high proportion of patients having risk factors of hypertension, hypercholesterolemia and a prior history of myocardial infarction (63\%) and none of the patients were diabetic by trial design. Mean total duration of clopidogrel use was $386 \pm 62$ days. All patients were also on stable aspirin and statin regimens (Table $1 \mathrm{~B}$ ). The type of stent implanted in the last procedure is listed in Table 2.

\section{Adverse clinical events}

There were no deaths, myocardial infarctions or stent thrombosis in this patient cohort after clopidogrel cessation. Two patients represented with symptoms, one with non-cardiac chest pain and another with angina symptoms, which did not require catheterization.

\section{Biomarker assessments}

Changes in inflammatory markers such as sCD40L, soluble P-selectin and hsCRP over 4 weeks after withdrawal of clopidogrel are shown in Table 3 (medians and interquartile ranges) and as mean percentage changes from baseline levels obtained at 12 months after clopidogrel administration. CD40L levels increased from a median of 224 to $324.5 \mathrm{pg} / \mathrm{ml}$ by week four after clopidogrel cessation (Table 3A).

P-selectin levels, on the other hand, appeared to increase slightly two weeks after clopidogrel cessation (from 44 to $50 \mathrm{ng} / \mathrm{ml} ; P<0.001)$ and then decreased to the baseline levels $(45 \mathrm{ng} / \mathrm{ml} ; P=0.488)$ after 4 weeks (Table $3 \mathrm{~B})$. Changes in soluble P-selectin levels correlated only weakly with changes in sCD40L levels (correlation coefficient reached 0.32 at 4 weeks with $P=0.024$; Fig. 2).

hsCRP levels decreased by $21 \%$ at 1 week $(P=0.008)$ and was still reduced by $18 \%$ by 4 weeks $(P=0.062)$ (Table 3C). 
Table 1 (A) Demographic baseline patient characteristics; (B) length of clopidogrel, aspirin and statin treatment

\begin{tabular}{ll}
\hline Variable & Total $(N=98)$ \\
\hline (A) Baseline characteristics & \\
Age (years) & \\
$\quad$ Mean & 63.3 \\
SD & 8.5 \\
Median (min, max) & $63(44,81)$ \\
Gender, $N$ (\%) & \\
Male/female & $78 / 20(79.6 / 20.4)$ \\
Race, $N$ (\%) & \\
Caucasian & $93(94.9)$ \\
Asian oriental & $5(5.1)$ \\
Risk factors/comorbidities, $N(\%)$ & \\
History of hypertension & $53(54.1)$ \\
Hypercholesterolemia & $80(81.6)$ \\
Congestive heart failure & $2(2.0)$ \\
Prior myocardial infarction & $62(63.3)$ \\
Atrial fibrillation & $3(3.1)$ \\
Stroke & $4(4.1)$ \\
Transient ischemic attack & $5(5.1)$ \\
Peripheral arterial disease & $8(8.2)$ \\
CABG & $6(6.1)$ \\
Peripheral angioplasty or bypass surgery & $1(1.0)$ \\
Current smoker & $16(16.3)$ \\
\hline Extent of exposure to clopidogrel & Total $(N=98)$ \\
and ASA and statins & \\
\hline & \\
\hline
\end{tabular}

(B) Time elapsed from initial clopidogrel administration

Clopidogrel use prior to withdrawal

$\begin{array}{ll}\text { Duration (days) } & 385.8 ; 62.4 \\ \text { Last dose (mg/day) } & 75.0 ; 0.0\end{array}$

ASA use prior to clopidogrel withdrawal

Duration (days)

$763.5 ; 1041$

Last dose (mg/day)

$101.2 ; 27.2$

Statin use prior to clopidogrel withdrawal

Duration (days)

$887.3 ; 1394$

Last dose (mg/day)

$43.1 ; 20.5$

Table 2 Type of stent implanted at index procedure

\begin{tabular}{lc}
\hline Type of DES & Total $(N=98)$ \\
\hline Implanted in most recent year, $N(\%)$ & \\
Paclitaxel eluting stent & $45(45.9)$ \\
Sirolimus eluting stent & $33(33.7)$ \\
Zotarolimus eluting stent & $18(18.4)$ \\
Everolimus eluting stent & $2(2.0)$ \\
Other type of stent & $6(6.1)$ \\
No stent implanted in most recent year & $4(4.1)$ \\
Type of stent implanted missing & $2(2.0)$ \\
\hline
\end{tabular}

sCD40L and stent type

Increase in CD40L varied with drug eluting stent used. Patients who were treated with zotarolimus stents appeared to have the greatest upregulation of sCD40L after clopidogrel discontinuation compared to the patients treated with paclitaxel, sirolimus eluting stents or bare metal stents (Fig. 3). The degree of sCD40L upregulation was dependent on baseline levels. There was no difference in total stent length between stent types implanted to confound the results.

\section{Discussion}

To our knowledge this is the first study to evaluate the effect of clopidogrel cessation at 1 year after initial stenting with drug eluting stents on inflammatory markers and markers of platelet activation in non-diabetic patients. This study was exploratory in nature, but nevertheless demonstrated a significant increase in CD40L expression after clopidogrel cessation indicative of possible enhanced platelet activation. Although not statistically significant at 4 weeks, P-selectin level increase seemed to parallel the CD40L changes, at least for the first 2 weeks, and correlated only weakly. P-selectin levels returned to baseline by 4 weeks suggesting that the stimulus causing this transient increase has disappeared. P-selectin is expressed upon platelet activation and binds the P-selectin glycoprotein ligand-1 on leukocytes [18]. P-selectin expression ultimately results in tissue factor expression by the monocytes and other inflammatory cytokines $[12,19]$. More general marker of inflammation hsCRP decreased by $21 \%$ at 1 week. This opposite effect suggests that the increase in sCD40L levels reflects solely the removal of the inhibitory effects of clopidogrel on platelet activity rather than an increase in pro-inflammatory state. The interpretation of this result is greatly limited by lack of measure of platelet inhibition or overall thrombogenicity assay (such as thromboelastography) [20-22].

While considerable data are now available on inflammatory markers and their modification with anti-platelet therapy in the context of acute coronary syndromes and percutaneous intervention [23, 24] it is not clear what impact cessation of an anti-platelet agent in a stable patient 1 year post-procedure has on inflammation and procoagulability. Even more difficult to interpret are confounding effects of drug eluting stent type. There may be a significant interaction between endothelial dysfunction due to local inflammation from the drug eluting stent polymer and platelet activation. Based on the animal data from Nakazawa et al. [25], the zotarolimus stent should have the greatest late loss and restenosis rate but also the best 
Table 3 Change in the levels of (A) sCD40L, (B) P-selectin and (C) hsCRP over 4 weeks presented as medians and interquartile ranges as well as mean percentage change

\begin{tabular}{|c|c|c|c|c|c|}
\hline & Baseline & Week 1 & Week 2 & Week 3 & Week 4 \\
\hline \multicolumn{6}{|c|}{ (A) sCD4OL levels over 4 weeks after clopidogrel cessation } \\
\hline \multicolumn{6}{|l|}{ CD40L } \\
\hline Median & 224 & 298 & 285.5 & 292 & 324.5 \\
\hline $\operatorname{Max}$ & 1450 & 2483 & 2559 & 2871 & 2474 \\
\hline Min & 10 & 36 & 23 & 42 & 18 \\
\hline Q3 & 364.5 & 478 & 569.5 & 509 & 541 \\
\hline Q1 & 129.5 & 161 & 160.75 & 155 & 199.5 \\
\hline Upper limit of normal & $0 \mathrm{pg} / \mathrm{ml}$ & & & & \\
\hline Lower limit of normal & $5000 \mathrm{pg} / \mathrm{nl}$ & & & & \\
\hline Average & 310.2 & 434.9 & 469.1 & 412.9 & 446.1 \\
\hline Std & 282.3 & 453.8 & 483.7 & 431.3 & 409.5 \\
\hline \multicolumn{2}{|l|}{ Mean percent change from baseline $+\mathrm{SE}$} & $\begin{array}{l}35 \%+10 \% \\
P<0.001\end{array}$ & $\begin{array}{l}39 \%+11 \% \\
P<0.001\end{array}$ & $\begin{array}{l}33 \%+10 \% \\
P<0.001\end{array}$ & $\begin{array}{l}39 \%+11 \% \\
P<0.001\end{array}$ \\
\hline \multicolumn{6}{|c|}{ (B) P-selectin levels over 4 weeks after clopidogrel cessation } \\
\hline \multicolumn{6}{|l|}{ P-selectin } \\
\hline Median & 44 & 49 & 50 & 45.5 & 45 \\
\hline Max & 104 & 114 & 138 & 147 & 150 \\
\hline Min & 20 & 22 & 9 & 17 & 23 \\
\hline Q3 & 58 & 60.25 & 60.25 & 59 & 55.25 \\
\hline Q1 & 37 & 40 & 39 & 35 & 35 \\
\hline Upper limit of normal & $51 \mathrm{ng} / \mathrm{ml}$ & & & & \\
\hline Lower limit of normal & $113 \mathrm{ng} / \mathrm{ml}$ & & & & \\
\hline Mean & 47.2 & 51.3 & 51.7 & 49.2 & 48.1 \\
\hline Std & 16.2 & 17.2 & 18.7 & 20.1 & 20.3 \\
\hline \multirow{2}{*}{\multicolumn{2}{|c|}{ Mean percent change from baseline $+\mathrm{SE}$}} & $9 \%+2 \%$ & $11 \%+2 \%$ & $4 \%+3 \%$ & $2 \%+3 \%$ \\
\hline & & $P<0.001$ & $P<0.001$ & $P=0.173$ & $P=0.488$ \\
\hline \multirow{2}{*}{\multicolumn{6}{|c|}{ (C) Levels of CRP over 4 weeks after clopidogrel cessation }} \\
\hline & & \multicolumn{4}{|c|}{ hsCRP (mg/L) } \\
\hline Median & 1.59 & 1.25 & 1.1 & 1.11 & 1.22 \\
\hline Min & 0.1 & 0.1 & 0.2 & 0.2 & 0.1 \\
\hline $\operatorname{Max}$ & 57.9 & 27.7 & 15.6 & 62.9 & 19.5 \\
\hline Q3 & 3.70 & 2.61 & 3.46 & 3.29 & 2.83 \\
\hline Q1 & 0.74 & 0.72 & 0.66 & 0.62 & 0.61 \\
\hline Average & 3.92 & 2.39 & 2.33 & 2.87 & 2.76 \\
\hline Std & 7.73 & 3.60 & 2.63 & 6.70 & 3.96 \\
\hline Lower limit of normal & $0 \mathrm{mg} / \mathrm{L}$ & & & & \\
\hline Upper limit of normal & $11 \mathrm{mg} / \mathrm{L}$ & & & & \\
\hline \multirow[t]{2}{*}{ Mean percent change from baseline $+\mathrm{SE}$} & & $-21 \%+7 \%$ & $-23 \%+7 \%$ & $-19 . \%+8 \%$ & $-18 \%+8 \%$ \\
\hline & & $P=0.008$ & $P=0.004$ & $P=0.038$ & $P=0.062$ \\
\hline
\end{tabular}

endothelialization and the least local inflammation compared to sirolimus and paclitaxel stents. These results in animal models were ascribed to better biocompatibility of phosphotylcholine polymer used in zotarolimus-eluting stents than polyethelyne co-vinyl acetate and poly-n-butyl methacrylate (sirolimus eluting stents) and polystyrene-bisobutyle-b-styrene (paclitaxel eluting stents). The latter two polymers have been shown to cause inflammation, local upregulation of tissue factor and macrophage activation [26]. We observed, however, the greatest sCD40L upregulation after clopidogrel discontinuation in patients treated with zotarolimus-eluting stents compared to sirolimus and paclitaxel drug eluting stents. This observation needs to be explored further in future studies and may 


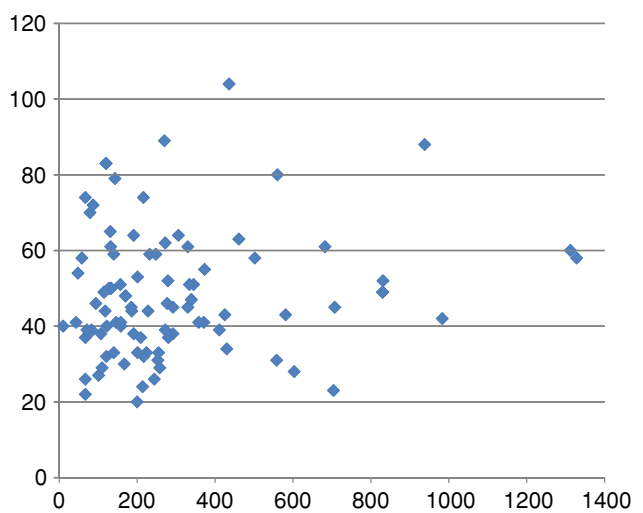

Spearman correlations between SCD40 Ligand and P-selectin

\begin{tabular}{lccccc}
\hline Visit & Baseline & Week 1 & Week 2 & Week 3 & Week 4 \\
\hline Correlation & 0.1034 & 0.2525 & 0.2323 & 0.2233 & 0.3183 \\
(P-value) (1) & $(0.3189)$ & $(0.0152)$ & $(0.0267)$ & $(0.0334)$ & $(0.0024)$ \\
Number of observation (N) & 95 & 92 & 91 & 91 & 89 \\
$(2)$ & & & & & \\
\hline
\end{tabular}

Fig. 2 Correlation between sCD40L and P-selectin levels

reflect a complex interaction between systemic proinflammatory state, platelet activation and local peri-stent polymer-induced inflammation.

Our results in this cohort of patients with stable coronary disease are in agreement with Azar et al. [27], who found only a modest decrease in SCD40L after starting clopidogrel in stable patients (17\% decrease after 8 weeks of therapy). It appears, however, that even at 12 months post-stenting clopidogrel still has an effect on inflammatory biomarkers and these effects are detectable in patients treated with stable doses of aspirin and statins. The poor correlation between SCD40L and P-selectin while somewhat perplexing, seem to echo the findings from the ELAPSE study on proinflammatory marker levels at 12 months after commencement of therapy [9]. Similarly, no correlation between more systemic hsCRP and more-platelet/atherosclerosis specific sCD40L/P-selectin was found in that study.

Lastly, a source of variability and the lack of strong correlations between different markers in our data set could be due to different doses of statins and potencies used in the study. Although a given patient had to be on a stable statin dose as the inclusion criteria, using variable doses with different down-modulation of the pro-inflammatory state (as reflected by hsCRP) could have made the patients on lower statin doses more likely to upregulate inflammatory markers after clopidogrel withdrawal. Ideally, when a larger study is performed all patients should be on a single statin such as rosuvastatin with LDL and hsCRP suppression goals optimized [28].

\section{Limitations}

Our study was limited by lack of biomarker level measurements before starting clopidogrel treatment as well as lack of concomitant data on platelet inhibition levels. The sample size was small and the study was exploratory in nature, and therefore not powered to detect clinical outcomes.

\section{Conclusions}

Patients treated with drug eluting stents at 1 year after implantation display significant increase in SCD40L and decrease in hsCRP after clopidogrel cessation. Further
Fig. 3 Change in SCD40L at 4 weeks after clopidogrel cessation in patients treated with different types of drug eluting stents. PES paclitaxel drug eluting stent; $S E S$ sirolimus eluting stent; $Z E S$ zotarolimus eluting stent; $O T H$ other; $B M S$ bare metal stent

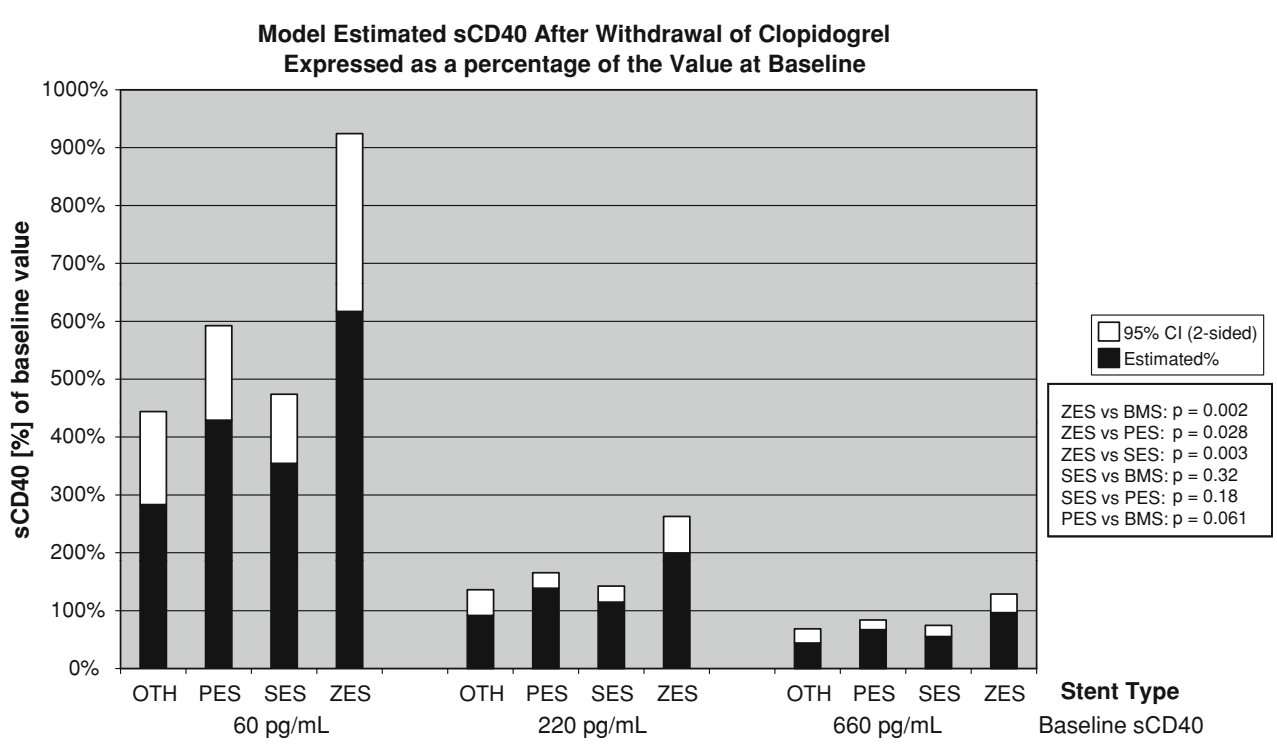


studies should elucidate if this increase in sCD40L levels reflects solely the removal of the inhibitory effects of clopidogrel on platelet activity or rather an increase in proinflammatory state. The latter hypothesis may be less likely given decrease in hsCRP levels. Zotarolimus stent implantation appears to be associated with the highest sCD40L upregulation after stopping clopidogrel but the clinical significance of this observation is uncertain.

Open Access This article is distributed under the terms of the Creative Commons Attribution Noncommercial License which permits any noncommercial use, distribution, and reproduction in any medium, provided the original author(s) and source are credited.

\section{References}

1. Steinhubl SR, Berger PB, Mann JT 3rd, Fry ET, De Lago A, Wilmer C et al (2002) Early and sustained dual oral antiplatelet therapy following percutaneous coronary intervention: a randomized controlled trial. JAMA 288(19):2411-2420. doi: 10.1001/jama.288.19.2411

2. Berger PB, Steinhubl S (2002) Clinical implications of percutaneous coronary intervention-clopidogrel in unstable angina to prevent recurrent events (PCI-CURE) study: a US perspective. Circulation 106(17):2284-2287. doi:10.1161/01.CIR.0000035924. 70846.20

3. Stettler C, Wandel S, Allemann S, Kastrati A, Morice MC, Schomig A et al (2007) Outcomes associated with drug-eluting and bare-metal stents: a collaborative network meta-analysis. Lancet 370(9591):937-948. doi:10.1016/S0140-6736(07)61444-5

4. Costa MA, Angiolillo DJ, Tannenbaum M, Driesman M, Chu A, Patterson J et al (2008) Impact of stent deployment procedural factors on long-term effectiveness and safety of sirolimus-eluting stents (final results of the multicenter prospective STLLR trial). Am J Cardiol 101(12):1704-1711. doi:10.1016/j.amjcard.2008. 02.053

5. Finn AV, Nakazawa G, Joner M, Kolodgie FD, Mont EK, Gold HK et al (2007) Vascular responses to drug eluting stents: importance of delayed healing. Arterioscler Thromb Vasc Biol 27(7):1500-1510. doi:10.1161/ATVBAHA.107.144220

6. Iakovou I, Schmidt T, Bonizzoni E, Ge L, Sangiorgi GM, Stankovic $\mathrm{G}$ et al (2005) Incidence, predictors, and outcome of thrombosis after successful implantation of drug-eluting stents. JAMA 293(17):2126-2130. doi:10.1001/jama.293.17.2126

7. Ho PM, Peterson ED, Wang L, Magid DJ, Fihn SD, Larsen GC et al (2008) Incidence of death and acute myocardial infarction associated with stopping clopidogrel after acute coronary syndrome. JAMA 299(5):532-539. doi:10.1001/jama.299.5.532

8. Angiolillo DJ, Fernandez-Ortiz A, Bernardo E, Ramirez C, Sabate M, Jimenez-Quevedo P et al (2006) Clopidogrel withdrawal is associated with proinflammatory and prothrombotic effects in patients with diabetes and coronary artery disease. Diabetes 55(3):780-784. doi:10.2337/diabetes.55.03.06.db051394

9. Saw J, Madsen EH, Chan S, Maurer-Spurej E (2008) The ELAPSE (Evaluation of Long-Term Clopidogrel Antiplatelet and Systemic Anti-Inflammatory Effects) study. J Am Coll Cardiol 52(23):1826-1833. doi:10.1016/j.jacc.2008.08.047

10. Angiolillo DJ, Costa MA, Shoemaker SB, Desai B, Bernardo E, Suzuki Y et al (2008) Functional effects of high clopidogrel maintenance dosing in patients with inadequate platelet inhibition on standard dose treatment. Am J Cardiol 101(4):440-445. doi: 10.1016/j.amjcard.2007.09.087
11. Biondi-Zoccai GG, Lotrionte M, Agostoni P, Abbate A, Fusaro M, Burzotta F et al (2006) A systematic review and meta-analysis on the hazards of discontinuing or not adhering to aspirin among 50,279 patients at risk for coronary artery disease. Eur Heart J 27(22):2667-2674. doi:10.1093/eurheartj/ehl334

12. Xiao Z, Theroux P (2004) Clopidogrel inhibits platelet-leukocyte interactions and thrombin receptor agonist peptide-induced platelet activation in patients with an acute coronary syndrome. $\mathrm{J}$ Am Coll Cardiol 43(11):1982-1988. doi:10.1016/j.jacc.2003. 10.071

13. Aggarwal A, Blum A, Schneider DJ, Sobel BE, Dauerman HL (2004) Soluble CD40 ligand is an early initiator of inflammation after coronary intervention. Coron Artery Dis 15(8):471-475. doi:10.1097/00019501-200412000-00003

14. Vivekananthan DP, Bhatt DL, Chew DP, Zidar FJ, Chan AW, Moliterno DJ et al (2004) Effect of clopidogrel pretreatment on periprocedural rise in C-reactive protein after percutaneous coronary intervention. Am J Cardiol 94(3):358-360. doi:10.1016/ j.amjcard.2004.04.035

15. Furman MI, Krueger LA, Linden MD, Fox ML, Ball SP, Barnard MR et al (2005) GPIIb-IIIa antagonists reduce thromboinflammatory processes in patients with acute coronary syndromes undergoing percutaneous coronary intervention. J Thromb Haemost 3(2):312-320. doi:10.1111/j.1538-7836.2005.01124.x

16. Nannizzi-Alaimo L, Alves VL, Phillips DR (2003) Inhibitory effects of glycoprotein IIb/IIIa antagonists and aspirin on the release of soluble CD40 ligand during platelet stimulation. Circulation 107(8):1123-1128. doi:10.1161/01.CIR.0000053559. 46158.AD

17. Heeschen C, Dimmeler S, Hamm CW, van den Brand MJ, Boersma E, Zeiher AM et al (2003) Soluble CD40 ligand in acute coronary syndromes. N Engl J Med 348(12):1104-1111. doi: 10.1056/NEJMoa022600

18. Rinder HM, Bonan JL, Rinder CS, Ault KA, Smith BR (1991) Dynamics of leukocyte-platelet adhesion in whole blood. Blood 78(7):1730-1737

19. Steinhubl SR, Badimon JJ, Bhatt DL, Herbert JM, Luscher TF (2007) Clinical evidence for anti-inflammatory effects of antiplatelet therapy in patients with atherothrombotic disease. Vasc Med 12(2):113-122. doi:10.1177/1358863X07077462

20. Bliden KP, DiChiara J, Tantry US, Bassi AK, Chaganti SK, Gurbel PA (2007) Increased risk in patients with high platelet aggregation receiving chronic clopidogrel therapy undergoing percutaneous coronary intervention: is the current antiplatelet therapy adequate? J Am Coll Cardiol 49(6):657-666. doi: 10.1016/j.jacc.2006.10.050

21. Gurbel PA, Bliden KP, Guyer K, Aggarwal N, Tantry US (2007) Delayed thrombin-induced platelet-fibrin clot generation by clopidogrel: a new dose-related effect demonstrated by thrombelastography in patients undergoing coronary artery stenting. Thromb Res 119(5):563-570. doi:10.1016/j.thromres.2006.05.006

22. Gurbel PA, Bliden KP, Guyer K, Cho PW, Zaman KA, Kreutz RP et al (2005) Platelet reactivity in patients and recurrent events post-stenting: results of the PREPARE POST-STENTING Study. J Am Coll Cardiol 46(10):1820-1826. doi:10.1016/j.jacc.2005. 07.041

23. Yip HK, Chang LT, Sun CK, Yang CH, Hung WC, Cheng CI et al (2006) Impact of clopidogrel on suppression of circulating levels of soluble CD40 ligand in patients with unstable angina undergoing coronary stenting. Am J Cardiol 97(2):192-194. doi: 10.1016/j.amjcard.2005.08.023

24. Gurbel PA, Bliden KP, Tantry US (2006) Effect of clopidogrel with and without eptifibatide on tumor necrosis factor-alpha and C-reactive protein release after elective stenting: results from the CLEAR PLATELETS 1b study. J Am Coll Cardiol 48(11):21862191. doi:10.1016/j.jacc.2005.12.084 
25. Nakazawa G, Finn AV, John MC, Kolodgie FD, Virmani R (2007) The significance of preclinical evaluation of sirolimus-, paclitaxel-, and zotarolimus-eluting stents. Am J Cardiol 100(8B):36M-44M. doi:10.1016/j.amjcard.2007.08.020

26. Luscher TF, Steffel J, Eberli FR, Joner M, Nakazawa G, Tanner FC et al (2007) Drug-eluting stent and coronary thrombosis: biological mechanisms and clinical implications. Circulation 115(8):10511058. doi:10.1161/CIRCULATIONAHA.106.675934
27. Azar RR, Kassab R, Zoghbi A, Aboujaoude S, El-Osta H, Ghorra P et al (2006) Effects of clopidogrel on soluble CD40 ligand and on high-sensitivity C-reactive protein in patients with stable coronary artery disease. Am Heart J 151(2):521 e1-521 e4

28. Ridker PM, Danielson E, Fonseca FA, Genest J, Gotto AM Jr, Kastelein JJ et al (2008) Rosuvastatin to prevent vascular events in men and women with elevated C-reactive protein. N Engl J Med 359(21):2195-2207. doi:10.1056/NEJMoa0807646 